\title{
Politique
}

\section{Tant d'amarres à larguer : une analyse des pratiques du mouvement des femmes}

\section{Claire Duguay et Micheline de Sève}

Numéro 5, hiver 1984

Femmes et pouvoir

URI : https://id.erudit.org/iderudit/040445ar

DOI : https://doi.org/10.7202/040445ar

Aller au sommaire du numéro

Éditeur(s)

Société québécoise de science politique

ISSN

0711-608X (imprimé)

1918-6584 (numérique)

Découvrir la revue

Citer cet article

Duguay, C. \& de Sève, M. (1984). Tant d'amarres à larguer : une analyse des pratiques du mouvement des femmes. Politique, (5), 51-73.

https://doi.org/10.7202/040445ar d'utilisation que vous pouvez consulter en ligne.

https://apropos.erudit.org/fr/usagers/politique-dutilisation/ 


\title{
Tant d'amarres à larguer: ${ }^{1}$ une analyse des pratiques du mouvement des femmes.
}

\author{
Claire Duguay \\ Micheline de Sève \\ Université du Québec à Montréal
}

Le féminisme a radicalisé une pluralité de femmes de tous les milieux, de tous les âges. L'ampleur du phénomène se mesure aux embarras qu'il suscite dans l'ensemble des organisations mixtes, de droite comme de gauche, où les femmes, parce que porteuses de changement, de questionnement, dérangent.

C'est ainsi que l'émergence objective d'une tendance autonome, celle des féministes, pose constamment problème dans des organisations qui contrôlent malaisément la parole des femmes et ne savent pas encore, en cas de conflit, où ces femmes nouvellement articulées de façon indépendante, vont se ranger.

Nous tenterons d'identifier dans cet article les mécanismes responsables des difficultés que rencontrent les féministes chaque fois qu'elles prétendent s'insérer collectivement dans des organisations mixtes. Nous nous efforcerons d'illustrer comment les structures en place freinent quasi automatiquement les tentatives

1. D'autres avant nous ont souhaité larguer les amarres mais l'histoire nous apprend que nos liens sont multiples et longs à trancher. Voir Sans fleurs ni couronnes, bilan des luttes et des rires, Montréal, édité par D.L.R., 1982, 104 et 122. 
d'expression autonome des femmes dont l'énergie s'épuise dans ce combat perpétuel contre une «machine» encore très bien rodée.

Ce diagnostic s'applique selon notre analyse aussi bien au mode de fonctionnement d'institutions politiques étatiques tel le comité des priorités du gouvernement du Parti québécois qu’à des organisations de gauche comme les centrales syndicales ou le Mouvement socialiste. En ce qui concerne celui-ci, l'existence de freins idéologiques et institutionnels est d'autant plus riche d'enseignements qu'il s'agit de la première organisation politique de gauche à se donner explicitement le mandat d'intégrer dans sa pratique comme dans son programme la reconnaissance de l'égalité entre les hommes et les femmes.

Nous nous attacherons à montrer d'autre part, comment les groupes autonomes de femmes, parce qu'autonomes précisément, ont rencontré moins d'obstacles pour défendre leurs revendications, ce qui ne les a pas empêchés de dépasser progressivement le niveau des luttes partielles pour se placer au coeur de la lutte contre les mentalités et les structures patriarcales à l'échelle de la société globale.

Nous avons choisi d'analyser certains mouvements de femmes qui de par leurs thèmes de ralliement, leurs modes de fonctionnement, leur style d'action concret, mobilisent «comme par enchantement» un grand nombre de personnes et contribuent à instaurer un type de militantisme plus souple et plus créateur. Nous avançons l'hypothèse que les groupes autonomes de femmes en particulier sont en voie d'implanter par leurs actions, leurs productions littéraires ou artistiques, leur humour, leur folie (?) une nouvelle culture politique qui traduit en termes socialement significatifs la richesse de l'expérience des femmes jusqu'ici confinée à la sphère de la vie privée. D'où l'émergence de thèmes et d'un style de lutte différents qui non seulement remettent radicalement en cause les rapports hommes-femmes partout où ils se posent mais démontrent comment ces rapports, loins d'être sectoriels, 
marquent toute l'organisation de la société. Aussi la présence des femmes s'affirme-t-elle à la fois sur le terrain de leurs revendications spécifiques et à l'intérieur de mouvements larges qui font appel à des valeurs fondamentales de la vie humaine: protection de la planète, respect de la nature, qualité de la vie, qualité de sa reproduction comme fonction économique essentielle, égalité des rapports humains, respect de la différence.

\section{Les mères font la bombe}

C'est dans ce cadre que nous interprétons la participation massive des femmes le jour de la fête des mères à une manifestation pour la paix et contre le nucléaire à Ottawa en mai dernier ${ }^{2}$. Par là elles ont renouvelé le sens d'une journée traditionnellement associée à une conception stéréotypée des fonctions maternelles. Elles ont affirmé la portée sociale de leur identité maternelle sans pour autant entrer dans les vues d'un gouvernement plus habitué à les associer à l'effort de guerre comme mères ou épouses de soldats ${ }^{3}$ qu'à entendre leurs protestations de citoyennes pacifistes. Elles ont marqué leur rupture avec une vision patriarcale de la maternité en retournant à leur avantage le potentiel mobilisateur d'une journée où les mères s'attendent à retenir l'attention publique et figurent à l'agenda des média. Ces mères ont ainsi donné un contenu différent à ces qualités qu'on leur reconnaît socialement: générosité, altruisme, dévouement, en démontrant que ces qualités réelles n'étaient pas en soi associées à la domination, à la passivité, à la vie privée mais pouvaient se traduire en un programme d'action politique tourné vers la vie et le respect de l'environnement.

2. Entrevue avec Danièle Lavoie, mère participante à cette manifestation, infirmière sociologue spécialisée en sociologie de la santé, féministe. Mai 1983. Environ 5000 femmes se sont retrouvées ce jour-là sur la colline parlementaire.

3. Geneviève Auger, Raymonde Lamothe, De la poêle à frire à la ligne de feu, Montréal, Boréal Express, 1981. 
Ce qui ne les a pas empêchées de publier un manifeste dénonçant la lutte entre les impérialismes concurrents ${ }^{4}$.

Il y a eu auparavant des exemples de manifestations de femmes en faveur de la paix ${ }^{5}$. Ce qui est nouveau dans ce type de manifestations pacifistes ou écologistes c'est que non seulement les femmes y sont présentes mais qu'elles portent visiblement ces mouvements sociaux. Des enfants, des jeunes, des personnes plus âgées, des artistes s'agglutinent autour d'elles pour composer un ensemble disparate, coloré, sans direction personnalisée et pourtant uni dans sa commune volonté de casser les modèles de rapports hiérarchisés qui marquaient, il n'y a pas si longtemps, jusqu'à nos défilés quasi militairement ordonnés, un contingent suivant l'autre avec un sérieux aussi imperturbable que des slogans répétitifs dénués de tout humour.

Ce qui frappe dans les occupations de terrain autour des centrales nucléaires, dans les marches joyeuses avec déguisements et fanfare, dans les rassemblements hétéroclites de gens de tout âge, de toutes conditions, c'est qu'ils illustrent un mode de vie en plus de témoigner d'une position politique claire et contestataire. L'alternative apparaît autant dans le discours que dans les transformations des comportements quotidiens et surtout dans le refus de la fragmentation des activités humaines. Ces gens qui mangent, parlent, dorment, dansent, chantent, vivent en un mot et dénoncent du même coup la stérilité des politiques de leurs gouvernements respectifs, rompent avec l'un des tabous les mieux intériorisés de nos sociétés, celui de la séparation du privé et du public. C'est par cette remontée à la surface des actes les plus simples et quotidiens de la vie des gens que se manifeste l'influence du principe de base du mouvement féministe voulant que le privé soit politique:

4. Tract diffusé à la manifestation contre le nucléaire à Ottawa, mai 1983, Mouvement écologiste et pacifiste.

5. M. Bilis, Socialistes et pacifistes, Paris, Syros, 1980. 
La personne et la société sont unies comme l'esprit et le corps. Tenter de définir laquelle est la plus importante des deux équivaut à déterminer ce qui de l'oxygène ou de l'hydrogène est le plus essentiel dans l'eau ${ }^{6}$.

Le mouvement féministe rompt avec les modèles de pouvoir hégémonique antérieurs, en cela que son extension se manifeste beaucoup moins sur le plan du discours et de la pénétration idéologique que de celui des comportements et d'une action subversive au sens fort du terme. Il ne s'agit plus de promettre des lendemains qui chantent mais de s'inscrire dans le présent et de transformer ici et maintenant des situations d'oppression immédiatement contraignantes. Le féminisme ne peut gérer l'abstraction sur le même mode que les mouvements sociaux qui l'ont précédé.

\section{Un exemple: les sage-femmes}

À travers le contrôle exercé sur leur corps et la négation de leur expérience par le pouvoir médical, les femmes vont de plus en plus prendre conscience qu'elles sont surmédicalisées quand il s'agit d'accoucher et qu'elles sont ignorées quand il s'agit d'avorter, de protéger leur santé au travail ou encore de réagir face à la violence qu'elles subissent. Cette prise de conscience va conduire entre autre à l'invention de nouvelles pratiques féministes d'autosanté et donc, à la prise en charge de leur corps par les femmes. Les sage-femmes présentent ainsi une alternative à la médecine patriarcale. Parce que la vie se donne sur un mode spécifique, qu'elle s'incarne dans le particulier et l'individuel, une approche féministe de l'accouchement suppose la maîtrise d'un savoir ouvert à des situations qui ne sont jamais tout à fait identiques ni comparables. Le savoir médical institutionnalisé réduit l'ensemble des cas hypothétiques à un certain nombre de pratiques codifiées et normalisées. Par opposition, la sage-femme intervient sur une

6. Marilyn Ferguson, Les enfants du verseau. Pour un nouveau paradigme, Paris, Calmann-Levy, 1981, 147. 
base individualisée qui vise à appuyer la femme enceinte dans la maîtrise personnelle de son accouchement ${ }^{7}$. La conception patriarcale de la médecine veut que la science du médecin et l'expérience de la sage-femme soient au mieux complémentaires. Ce qui se traduit par la subordination d'un savoir féminin au savoir institutionnalisé dominant. Encore cette tolérance ne va-t-elle pas de soi, témoin, malgré les revendications contraires de groupes de femmes ${ }^{8}$, la centralisation des soins obstétriques dans quelques hôpitaux spécialisés plutôt que la multiplication des chambres de naissance ou encore l'acceptation de l'accouchement à la maison.

L'affrontement politique qui en résulte sort des formes classiques de rapports entre groupes de pression. Si les médecins sont constitués en corporation, c'est à titre individuel que les sagesfemmes s'opposent à eux, les confrontant chaque fois en rapport avec un cas particulier. La force des sages-femmes ne tient pas à celle de leur corporation professionnelle mais à la solidarité des femmes qui ont vécu une expérience unique grâce à leur soutien et à la mobilisation des autres qui se rendent compte qu'elles ont été dépossédées de leur capacité à ce niveau par l'intermédiaire d'un pouvoir médical aveugle à la dimension personnelle de chaque accouchement et à la capacité de contrôle des femmes de leur corps $^{9}$.

\section{Un mouvement kaléidoscopique}

Solidaire dans certaines de ses luttes, le mouvement des femmes n'est pas univoque pour autant. Certaines persistent à identifier le féminisme en terme d'intégration aux institutions

7. Sylvie Van Brabant, Serge Grégoire, Louise Dugal, Depuis que le monde est monde, Dossier d'accompagnement d'un film produit par Naissance-Renaissance, 1981.

8. Rapport-synthèse, Accoucher ou se faire accoucher, Colloques régionaux de l'ASPQ, Bulletin d'octobre 1981.

9. B. Ehrenreich, D. English, Des experts et des femmes, 150 ans de conseils prodigués aux femmes, Montréal, Les Éditions du Remue-Ménage, 1982. 
sociales existantes croyant résoudre la question des femmes par une simple transformation comptable des règles de répartition sexuelle des postes électifs ${ }^{10}$. D'autres, sensibles à la capacité des structures politiques de mouler les individus hommes ou femmes selon des modèles pré-déterminés, se rendent compte que pour être acceptées au sein d'organisations de ce type, il faut passer par un processus d'apprentissage et de socialisation qui implique l'abandon, ou du moins la secondarisation de ses objectifs spécifiques initiaux au profit de la "compréhension des intérêts supérieurs» du groupe d'accueil ${ }^{11}$.

\section{Le prix d'une servitude}

C'est ainsi qu'en l'absence de structures de soutien, les femmes isolées qui entreprennent de se manifester publiquement de la sorte subissent un processus d'érosion de leur personnalité, de leurs besoins, de leur condition propre.

Ainsi les femmes qui participent activement aux divers mouvements politiques sont, souvent, anti-féministes. Fières d'avoir réussi à se faire accepter dans un milieu d'hommes, le rappel de l'oppression de la femme les blesse car elles veulent se croire libres. De la même manière, le métis accepté dans la société blanche renie souvent son origine de couleur et ne veut pas se sentir concerné par le racisme ${ }^{12}$.

Loin de porter les revendications des femmes à l'intérieur des partis politiques, les nouvelles femmes politiques servent plus souvent de soutien et d'éléments modérateurs auprès de leurs consoeurs. Si Lise Payette avait été moins isolée, n'aurait-elle pas

10. À l'exemple des quota en France fixant à $75 \%$ maximum l'inscription de personnes du même sexe sur chaque liste de candidats aux élections municipales. Ce projet de loi a d'abord été adopté à l'Assemblée nationale en 1982 pour être ensuite rejeté par le Conseil constitutionnel. Voir le Monde du 20 août 1982 et des 6-7 mars 1983.

11. Voir là-dessus, la réflexion lucide d'Huguette Bouchardeau, secrétaire nationale du PSU, Un coin dans leur monde, Paris, Ed. Syros, 1980.

12. Anne et Jacqueline, "D'un groupe à l'autre» in Partisans, Libération des femmes, Paris, Maspero, 1974, 170-171. 
rencontré les mêmes obstacles dans la mécanique du pouvoir figée qui s'est chargée de la mettre au pas? L'exemple récent de Pauline Marois, pourtant entourée d'une équipe plus considérable, témoigne de la précarité du statut des femmes à l'intérieur de structures promptes à gommer leur spécificité. Que René Lévesque ait cru dorer la pilule en la nommant vice-présidente du Comité des finances au moment de l'évincer du Comité des priorités à titre de ministre de la Condition féminine illustre bien le genre de piège qui attend les femmes au sein des institutions politiques traditionnelles. Où commencent, où s'arrêtent les compromissions? Comment justifier l'appui de la ministre de la Condition féminine à des politiques gouvernementales nettement en recul par rapport à des revendications qu'elle-même portait auparavant en matière de services de garde ou de subventions à des groupes de femmes? Recourir à l'explication par la crise ou à l'insuffisance des pouvoirs du gouvernement provincial par rapport au gouvernement fédéral montre bien l'effet récupérateur du discours du pouvoir sur les prétentions de défense des intérêts d'un groupe spécifique. Ce type de choix politique mène à une impasse: ou la démission à plus ou moins brève échéance ou l'adoption de pratiques de jour en jour plus éloignées du projet initial. En ce sens, le cas québécois n'est pas un cas isolé. Eleanore Smeal, présidente du NOW (Mouvement américain pour la défense du droit des femmes), déclarait dans un entretien avec Betty Friedan: «On ne peut pas s'attendre à ce qu'une femme, quelle qu'elle soit, une fois investie d'un quelconque pouvoir, ne vende pas les autres femmes à bas prix» ${ }^{13}$.

\section{Le féminisme est-il syndicable?}

L'introduction de structures autonomes de femmes peut compenser dans une certaine mesure le poids des structures instituées

13. Betty Friedan, Le second souffle, Paris, Hachette, 1982, 22. 
et permettre la formulation de revendications propres aux femmes. L'exemple des comités de condition féminine des centrales syndicales marque un progrès puisqu'ils ont permis de contrer la dispersion des énergies et d'articuler avec plus de force les positions des femmes à la fois sur le terrain de leurs revendications et quant à la reformulation des conditions concrètes d'exercice de la démocratie. Sans ces structures semi-autonomes, les revendications des syndiquées sur l'égalité salariale, les congés de maternité, les congés parentaux, les garderies n'auraient pas connu l'évolution que l'on sait. D'autant plus que leur acceptation ne s'est pas faite sans heurt.

Quant à la légende de la faible tendance à la participation des femmes, de récentes enquêtes aussi bien des comités de condition féminine de la CSN que de la $\mathrm{CEQ}^{14}$ se sont chargées de démontrer qu'elle n'était pas une caractéristique naturelle de la population syndicale féminine mais qu'elle était bien plutôt attribuable à un style de militantisme «musclé» étranger à la réalité matérielle du mode de vie et de pensée des femmes. Hélène Massé, retraçant dans son mémoire de maîtrise la mise sur pied des syndicats d'enseignantes rurales au cours des années 1930 a montré comment les femmes syndiquées s'organisaient de manière plus collégiale et savaient rester proches de l'ensemble des préoccupations quotidiennes des institutrices rurales ${ }^{15}$.

Ce mode de militantisme se traduisait par une participation massive des femmes aussi bien à leurs assemblées syndicales qu'à la rédaction de leur bulletin, La (Notre) Petite Feuille où tous les sujets étaient abordés tant par la direction que par de simples membres. En un an, après la fusion entre la Fédération catholique

14. Rapport du comité de la condition féminine de la CSN, Les femmes à la C.S.N. n'ont pas les moyens de reculer, congrès de la CSN, Québec, mai 1982.

15. Hélène Massé, De l'autonomie d'action syndicale des femmes à la récupération patriarcale, une étude de cas: la Fédération catbolique des institutrices rurales de 1936 à 1953, Québec, Université Laval, thèse de maîtrise, 1982. 
des institutrices rurales et les autres corporations d'enseignants ruraux et urbains, elles qui s'exprimaient à $96 \%$ dans leur journal ne le feront plus qu'à $26,8 \%$ dans le nouvel organe de diffusion de la Corporation des instituteurs et institutrices catholiques L'enseignement, journal plus spécialisé et majoritairement contrôlé par la direction masculine de la fédération qui deviendra plus tard la $\mathrm{CEQ}^{16}$. La prise du pouvoir par les instituteurs ruraux et surtout urbains entraîne alors une large démobilisation des enseignantes syndiquées: «Les institutrices rurales ne se sentant pas écoutées, comprises et défendues par la corporation vont se désolidariser du mouvement et quitter ses rangs en grand nombre ${ }^{17}$. C'est aux femmes des comités de condition féminine que l'on doit d'avoir repris la critique des méthodes centralistes de direction et d'avoir ouvert le débat sur les conditions d'exercice de la démocratie syndicale ${ }^{18}$.

Cette aptitude des femmes syndiquées à pratiquer le décloisonnement de leurs rôles de syndiquées, d'enseignantes, de travailleuses ou d'usagères de la santé, de parentes, leur a permis de formuler une problématique lors de la dernière négociation du front commun qui tranchait nettement avec la pratique stricte de défense des intérêts immédiats des seuls syndiqués. C'est ainsi que les femmes du comité de condition féminine de la CSN ont formulé dans un document «Nous aussi, nos enfants sont à la maison» une plate-forme visant à traduire le lien entre leurs luttes et celles de l'ensemble des femmes impliquées comme travailleuses, usagères des services sociaux, ménagères et appelées ainsi à subir aussi bien le poids des grèves que celui des coupures dans le

16. Ibid., 171.

17. Ibid., 173

18. Voir l'analyse du syndicalisme au masculin, dans Les femmes a la CSN niont pas les moyens de reculer. op. cit., Voir aussi l'intervention d'un groupe de femmes au dernier congrès de la CEQ "Nous aurons les chefs qu'ils toudront", reprise dans une entrevue de la Vie en Rose "La longue marche des filles de syndicats", La V'ie en Rosi', septembre-octobre $1982,41$. 
budget des politiques sociales ${ }^{19}$. Malgré la perspective très mobilisatrice de ce document pour regagner la faveur de l'ensemble des citoyens qu'une revendication polarisée par la défense unique de la sécurité d'emploi pouvait difficilement séduire dans une conjoncture de crise, cette orientation n'a pas été retenue par les dirigeants syndicaux. La tournée prévue pour diffuser cette approche alternative de la lutte syndicale dans les différents comités de condition féminine a été annulée au nom de l'urgence de la mobilisation des effectifs pour les fins internes de ralliement des militant(e)s de la Fédération des affaires sociales (FAS) appelée à prendre un nouveau vote de grève. Cet épisode récent du traitement de la perspective amenée par le comité de la condition féminine de la CSN montre bien comment fonctionne le mécanisme de secondarisation du point de vue des femmes, même et surtout en période de lutte intense. Leurs revendications ne sont jamais vues comme prioritaires y compris dans le cas où ce sont les femmes qui. subissent majoritairement le poids des attaques des patrons ou de l'État comme c'était le cas dans cette lutte du Front commun.

Il faut dire que nombre de femmes acceptent d'autant plus facilement d'épouser les vues de leurs dirigeants sur les stratégies adéquates que ceux-ci jouissent du prestige associé à leur longue expérience de pratique de luttes éprouvées, expérimentées en dehors d'elles. La légitimité syndicale s'est construite officiellement sans elles et ne laisse guère place à leur initiative qui rencontre de fortes résistances aussi bien idéologiques - la fameuse loyauté syndicale - qu'organisationnelles - le cadre juridique de fonctionnement externe et interne. Ajoutons que quand ce ne sont pas les dirigeants syndicaux qui freinent l'action des comités de condition féminine, c'est l'État qui s'en charge. Témoin la fin de non recevoir opposée à la volonté des syndicats d'introduire les

19. Texte reproduit sous ce titre dans les Cabiers du Socialisme, nos 12-13, 1983, 325-336. 
travailleuses et travailleurs des garderies au nombre des participants à la table de négociation du secteur de la FAS.

Semi-autonomie, disions-nous de la structure des comités de condition féminine. Non seulement parce que structurellement ils restent subordonnés aux instances de direction de leurs centrales respectives, mais surtout parce que la définition des objectifs de ce type d'organisme permet constamment de justifier la secondarisation des revendications des femmes au nom d'impératifs plus larges. Les quelques féministes qui survivent à ce régime de minorisation et d'infériorisation systématiques voient leur énergie minée par les constants délais que subissent leurs positions jugées partielles quand elles sont directement reliées à la spécificité de la condition des femmes ou utopiques quand elles osent formuler d'un point de vue féministe «anti patriarcal» une analyse globale des divers problèmes qui se posent.

\section{Une nouvelle forme d'obéissance: la subordination}

On a pensé, jusqu'à tout récemment, que l'obtention de struçtures autonomes de femmes au sein d'organisations politiques règlerait "la question des femmes». L'expérience du Mouvement socialiste, mouvement pour le socialisme, l'indépendance, la démocratie et pour l'égalité des rapports entre les hommes et les femmes s'est chargée de nous détromper là-dessus ${ }^{20}$. Les femmes y ont gagné de haute lutte, leur droit de former des caucus de

20. Pour un plus grand souci d'objectivité (à noter cependant que l'objectivité n'est pas la neutralité), nous tenons à préciser qu'une des rédactrices de cet article, Claire Duguay, a été membre du Mouvement socialiste depuis ses débuts et qu'elle fait partie du groupe des 14 démissionnaires du comité des femmes de la région de Montréal. Nous ne voulons pas reprendre ici le vieux débat introduit par Max Weber mais pour une approche nouvelle voir Huguette Dagenais: "Quand la sociologie devient action: l'impact du féminisme sur la pratique sociologique», Dominique Gaucher, France Laurendeau, LouiseHélène Trottier: "Parler de la vie: l'apport des femmes à la sociologie de la santé" in Sociologie et Sociétés, vol. XIII, no 2, octobre 1981. 
femmes, le droit de se réunir en assemblée générale, le droit à leurs structures autonomes (comités régionaux de femmes, comité national des femmes socialistes), le droit d'utiliser leur propre micro en alternance avec celui de leurs camarades masculins. Les femmes y ont gagné de pouvoir acheminer leurs revendications et leurs critiques en toute quiétude... Mais cela n'a pas empêché que leur parole continue d'être perçue par un grand nombre comme l'expression de revendications partielles ou l'effet de leur identité socio-culturelle plutôt que comme une expression démocratique de type nouveau ${ }^{21}$. En demandant que leur spécificité soit reconnue, les féministes ne demandaient pas l'ajout de quelques amendements à la plate-forme du groupe mais exigeaient la transformation de la façon même de concevoir l'élaboration d'un point de vue global sur l'ensemble des réalités concrètes. Le débat sur la loi 111 et la lutte du front commun qui a débouché pour elles sur une critique de la division et de l'organisation du travail témoignent de la fécondité de ce nouveau mode d'analyse des rapports sociaux ${ }^{22}$. Les femmes y ont compris que les coupures actuelles dans les politiques de santé et d'éducation non seulement remettaient en question leur droit au travail social mais conduisaient - par la reprivatisation des services sociaux - à un accroissement de leurs tâches ménagères. Solidaires entre elles, travailleuses salariées et ménagères ont aussi lié leurs revendications à celles d'autres catégories sociales affectées par la crise. Et de fait, le rapport d'oppression qu'elles subissent se répercute sur tous ceux, hommes ou femmes, chômeurs ou déclassés qui, faute de santé

21. Le texte de Raymond Laliberté, "L'actualité de l'alternative sacialiste au Québec", présenté au colloque de la société québécoise de sciences politique, à paraître dans les Actes du congrès de l'ACFAS, Trois-Rivières, mai 1983, traduit bien cette conception du féminisme.

22. Voir le texte, "La double journée de travail au coeur du débat", présenté par le comité des femmes de la région de Montréal dans le cadre du débat organisé par le Mouvement socialiste sur la lutte du front commun et la loi 111, mars 1983. 
ou de moyens financiers perdent leur autonomie pour se retrouver condamnés à la dépendance et à la passivité, qu'il s'agisse de tomber sous la coupe de l'État ou sous celle de leur famille.

Les structures autonomes ont permis la reconnaissance de la «présence» des femmes à part entière au sein du Mouvement socialiste mais la bataille de la reconnaissance de la légitimité du féminisme comme théorie politique et mouvement social est loin d'être gagnée. L'expression de points de vue féministes est constamment renvoyée à des considérations d'un autre ordre. Ce que les femmes disent n'est pas écouté comme tel mais interprété politiquement comme faisant le jeu de telle ou telle tendance; écourté pour faire place à des débats soi-disant plus urgents et importants; ou carrément discrédité comme l'illustration d'un manque flagrant d'expérience politique.

Ainsi le mandat de l'assemblée générale des femmes de la région de Montréal concernant la convocation d'une assemblée sur la démocratie à l'intérieur du mouvement en avril dernier suite aux malaises ressentis par beaucoup de militantes et de militants a été redéfini par la direction du comité régional de Montréal à travers le prisme de la «menace» représentée par la présence des ex-militants de Combat socialiste (tendance trotskyste) à l'intérieur du mouvement. La volonté des femmes de poser ce débat à partir des besoins ressentis par l'ensemble des militant(e)s a été ici ramenée à un affrontement de tendances et vidée de toute portée critique large.

La démission collective de 14 membres du comité régional des femmes de Montréal au terme de ce balayage sur la question des tendances organisées à l'intérieur du mouvement socialiste marque non seulement un refus clair de ce type de débat et de l'anti-démocratisme dans lequel il s'est déroulé mais intervient principalement parce que la parole politique des féministes n'a jamais été prise en considération de façon autonome malgré la 
publication de deux textes exprimant leur point de vue sur leurs propres malaises et diffusés aux différentes instances ${ }^{23}$.

Constamment, parce que ces féministes se démarquaient de la direction actuelle du comité régional de Montréal, et parce qu'il y avait au sein du comité des femmes des militantes d'exCombat socialiste, la parole politique du comité des femmes de la région de Montréal a été associée, puis subordonnée elle aussi à la présence des Trotskystes. Le comité des femmes de la région de Montréal non seulement refusait les termes du débat qui ne pouvaient que conduire à une impasse mais voulait exprimer dans sa propre pratique comment avait été vécue cette question des tendances. Si la direction du mouvement socialiste voulait débattre cette question, elle devait envisager toutes les tendances organisées. Le débat sur les structures autonomes de femmes avait permis au comité d'être confronté à une tendance syndicale dans la région de Montréal qui s'était opposée massivement à l'existence de ces structures autonomes sous prétexte qu'elles étaient appuyées par les militant(e)s d'ex-Combat socialiste. Nombre de féministes n'acceptaient plus de secondariser la question des femmes sous prétexte qu'il y avait une menace supérieure dans le mouvement, c'est-à-dire la présence d'une tendance trotskyste. La menace pour le comité n'était pas la même. La menace, c'était de voir battre les structures autonomes de femmes parce qu'elles étaient la condition nécessaire à leur place dans le mouvement. Le comité des femmes était moins préoccupé de la présence de points de vue différents à l'intérieur du mouvement, que de développer à partir des faits, des luttes, une dynamique qui permette des débats

23. Voir les textes internes du Mouvement socialiste: «Position du comité des femmes de la région de Montréal sur la démocratie dans le mouvement", mai 1983. "On est tannées de se faire crier des noms", texte de démission de 14 membres du comité des femmes de Montréal présenté au Conseil national, juin 1983. - Voir aussi la réponse aux démissionnaires de Jody Freeman et Michèle Berthelot, «Démissionnerr ou rester là?», juillet 1983 . 
démocratiques ouverts dans la construction d'un modèle socialiste à inventer où la question des femmes serait, elle aussi, prioritaire.

Il importe de souligner que dans ce type d'organisation mixte, les féministes ont à défendre non seulement l'autonomie de leurs structures et de leurs positions par rapport à leurs camarades masculins mais qu'elles sont constamment confrontées à d'autres femmes moins détachées qu'elles de l'emprise des idéaux d'unité abstraite qui aboutissent à tout coup à nier leurs aspirations propres. Chaque fois qu'un affrontement risque de se produire, ces femmes appellent leurs consoeurs à taire des différences jugées «secondaires» ou à reporter à plus tard leurs propositions au nom de la compréhension des intérêts «supérieurs» de l'organisation quand ce n'est pas par le sempiternel renvoi à la nature du contexte. C'est ainsi que des féministes sont constamment accusées de manquer d'expérience ou de sens tactique, ou si cela ne suffit pas, qualifiées d'extrémistes radicales ${ }^{24}$.

Certes le long combat pour la reconnaissance «idéologique» du féminisme est résolument engagé en particulier au sein des organisations de gauche mais on aurait tort de minimiser la force des habitudes, le poids des traditions, le style de fonctionnement et de langage dominants qui font que les féministes continuent d'apparaître marginales, étrangères dans un monde régi sur le mode patriarcal. Elles se retrouvent constamment sur la défensive, vidées à mesure de leurs énergies dans un combat où l'ennemi est à la fois partout parce qu'elles ne sont pas sur leur terrain et nulle part parce que d'autres thèmes sont toujours plus importants à considérer que ceux qu'elles tentent de soulever:

L'erreur fondamentale consiste à vouloir négocier l'alliance avant même d'exister en tant que force politique organisée et d'avoir établi un véritable rapport de force. Or en politique on ne respecte que les forts. Les minorités - fussent-elles mâles - ne sont pas respectées dans le camp patriarcal. Dispersées dans les organisations patriarcales, nous serons toujours minoritaires, parce que la plus intelligente des

24. Jody Freeman, Michèle Berthelot; op. cit. 
politiques passe par des êtres sexués et ne reste pas au ciel des idéologies abstraites, qu'elles soient socialiste, communiste, autogestionnaire ou même écologiste. Les hommes de toutes les familles politiques ont depuis toujours maintenu les femmes en état de mineures et ne renonceront pas à cette tutelle qu'ils exercent depuis des milliers d'années. Ils n'ont jamais pu se résoudre, dans l'histoire, à une autre attitude que paternaliste vis-à-vis de nous dans le meilleur des $\operatorname{cas}^{25}$.

\section{Globaliser sans niveler}

L'implantation des féministes dans les organisations larges, politiques ou autres, traduit une volonté positive de lier la lutte des femmes à une transformation globale de la société. Conscientes des limites des luttes sectorielles dans la santé ou contre toutes les formes de violence faites aux femmes, les féministes qui se sont d'abord identifiées à la gauche se sont heurtées les unes après les autres, que cela soit à «En lutte», au PCO, au mouvement socialiste, à la résistance opiniâtre des structures et de l'idéologie patriarcale.

D'un autre côté, les féministes qui sont restées sur leur propre terrain et qui ont continué à lutter sur des thèmes dits sectoriels: santé, sexisme, pornographie, viol, etc... ont débouché progressivement sur une critique globale de la société. À partir de leur appréhension des réalités concrètes, elles ont nommé le patriarcat, cette structure de domination sexuelle et sociale qui fragmente et hiérarchise l'ensemble des pratiques sociales ${ }^{26}$.

Alors que la gauche continue de méconnaître le sens politique de la lutte contre la pornographie par exemple, les groupes autonomes de femmes ont produit à partir de leurs actions, une analyse théorique de ce phénomène en terme de sexisme et d'attaque

25. Suzanne Blaise, Des femmes de nulle part ou le préféminisme politique. Paris, Ed. Tierce, 1981, 156.

26. Voir les bilans récents de différents groupes autonomes de femmes: Véronique O'Leary, Louise Toupin, Québécoises deboutte, Montréal, Ed. du Remue-Ménage, 1983 , tomes I et II.

- Les Têtes de pioche, journal des femmes, Montréal, Ed. du Remue-Ménage, 1980.

- Sans fleurs ni couronnes, bilan des luttes et des rires, Montréal, édité par DLR, 1982. 
haineuse pour ravaler la femme au rang d'objet et nier sa personnalité ${ }^{27}$. Elles ont démontré le lien entre l'affirmation des femmes sur la scène publique et l'offensive de propagande répressive que constituent l'encouragement à la violence dans les rapports sexuels et la diffusion massive de ces images de femmes violées, attachées, battues, brûlées ou même tuées comme des bêtes soumises ou asservies par leurs maîtres. La gauche persiste encore à réduire ce phénomène à l'aspect culturel, l'analysant en termes de liberté d'expression ou de conception plus ou moins laxiste de l'érotisme et des «jeux» amoureux alors qu'il s'agit d'une industrie qui touche les aspects les plus fondamentaux des rapports inter-ethniques ou inter-classes. Le mépris de la femme est le modèle de base qui fonde la négation de l'humanité du Noir, du Colonisé, du Juif ou de l'étranger en général. C'est ainsi qu'à propos de la pornographie et du sexisme sous toutes ses formes, les féministes ont développé une analyse matérialiste du rapport au corps ${ }^{28}$. Elles ont ainsi poussé d'un cran la compréhension théorique des fondements de l'exploitation économique qui dans le cas des femmes, des immigrants ou des jeunes, s'appuie sur leur identité soi-disant biologiquement inférieure de non-adultes, non-blancs, non-autochtones, ou non-mâles pour déterminer leur affectation à des positions sociales inférieures qui «justifient» leur oppression d'abord, leur surexploitation ensuite.

Ainsi, la travailleuse en butte au harcèlement sexuel d'un camarade de travail(!), et dont le syndicat défend son assaillant ne peut pas accepter de retirer sa plainte au nom de la solidarité syndicale. Elle sait qu'il ne s'agit pas d'un accident de parcours mais que ce type de comportement l'atteint aussi profondément que la ségrégation dont sont victimes les immigrants y compris leurs descendants. Comment pourrait-elle admettre qu'elle doive

27. Laura Lederer, éd., L'Envers de la nuit, les femmes contre la pornographie, Montréal, Ed. du Remue-Ménage, 1983.

28. Ibid. 
être solidaire d'un travailleur mis-à-pied sans préavis alors que son syndicat ne l'a même pas défendue quand elle-même était en butte à une attaque injustifiée, discriminatoire? ${ }^{29}$

Réduire le harcèlement sexuel à un écart de conduite sans gravité tout au plus moralement condamnable, c'est recourir à un type d'explication qui a longtemps permis aux associations patronales de justifier nombre de vexations contre des ouvriers qualifiés de paresseux, de fourbes et d'ignorants à qui il n'était pas question non seulement de payer un salaire décent mais de leur reconnaître le droit à la dignité. Les femmes conscientes de leur oppression ne peuvent plus faire de compromis là-dessus, pas plus qu'un ouvrier qui a pris conscience de son exploitation ne pourra fraterniser avec son patron. En ce sens, les organisations féministes non mixtes sont au mouvement des femmes ce que les syndicats ont été à l'origine pour le mouvement ouvrier: elles sont la condition de la possibilité même d'une lutte efficace contre le patriarcat.

Ce qui caractérise le mouvement des femmes c'est que même si ses luttes ont une portée idéologique certaine, ce n'est pas tant sur le discours qu'il s'appuie pour défendre ses positions mais sur des manifestations et des actions qui démontrent concrètement comment s'y prendre pour satisfaire les besoins exprimés par les femmes. Ainsi la pratique du Centre des femmes au début des années 1970 représente un bel exemple de cette orientation ${ }^{30}$. Des femmes de toutes les régions du Québec étaient reçues, hébergées, aidées afin que leur avortement se passe dans les meilleures conditions humaines et médicales. L'implantation de ce centre correspondait non seulement à une nécessité urgente mais démontrait la force de la solidarité des femmes quand elles s'attaquent à la

29. Voir «Le harcèlement sexuel à l'école», in Madame au Foyer, juin 1983. À venir dans Châtelaine, les résultats de l'enquête effectuée par le YWCA et la Vie en Rose sur le harcèlement sexuel. Bouleversant.

30. Véronique O'Leary et Louise Toupin, Québécoises deboutte, op. cit. 
résolution de leurs propres problèmes. L'existence même de ce centre défiait l'État, les juges et la police, et la pression des femmes obligeait différents organismes populaires et syndicaux à entrer dans l'arène pour défendre le droit des femmes au contrôle de leur corps. Les femmes imposèrent ainsi la révision des politiques en matière d'avortements thérapeutiques. L'État fut forcé de produire un discours et de s'engager dans un certain nombre de pratiques de gestion ${ }^{31}$ à propos d'un secteur éminemment conflictuel. La répression et le silence ne pouvaient plus suffire à réduire la résistance des femmes qui entendaient en finir avec des avortements clandestins et la honte attachée au choix de la maternité volontaire. Cet affrontement eut pour effet de révéler au grand jour la signification de l'identité patriarcale de l'État comme de ses répondants dans la société civile et de tous les organismes de défense de la «cellule de base naturelle» de la société: la famille ${ }^{32}$.

Cet exemple illustre bien le mode de fonctionnement politique des femmes. Partir de leurs propres besoins, prendre elles-mêmes l'initiative de mettre en place les moyens de les résoudre sans attendre, ni même demander l'intervention de l'État dont elles savent qu'il est étranger, sinon hostile à leurs propres réalités.

Il est de plus à remarquer que les femmes laissées libres de s'organiser entre elles échappent rapidement au conformisme du modèle social imposé aux femmes. L'exemple de l'AFEAS est significatif à ce niveau. Ce groupe réputé conservateur ne s'est-il pas engagé dans un processus de défense de l'autonomie financière des épouses collaboratrices de leur mari qui rompt avec une conception traditionnelle du dévouement aveugle et inconditionnel des épouses des petits producteurs en milieu rural et urbain? C'est d'elles que sont venues les premières propositions concrètes pour

31. La mise sur pied des cliniques Lazure illustre bien la nature de la gestion étatique quand il s'agit de l'avortement visant le contrôle par l'État du corps des femmes.

32. "La vie des femmes n'est pas un principe", des groupes de femmes répliquent à l'épiscopat, le Devoir, 11 décembre 1981. 
en finir avec la gratuité du travail domestique dans un secteurclé de l'économie. De même que le débat sur l'avortement a engagé une série d'actions sur l'ensemble des activités liées au choix de la maternité comme de la non-maternité, de même, les revendications des femmes de l'AFEAS ou des cercles de fermières sont susceptibles d'engendrer la révision de l'ensemble du cadre des activités liées à l'économie domestique.

Ce mode d'intervention des groupes autonomes de femmes, féministes ou non, sur leur propre terrain et en marge des institutions politiques étatiques a donné jusqu'ici de meilleurs résultats pour l'amélioration des conditions de vie et de travail des femmes que toute autre forme d'intégration à des organismes socio-politiques mixtes même de gauche. Cependant la dispersion de ces groupes, l'absence relative de lien de communication entre des femmes engagées dans divers champs de pratique ne cessent d'inquiéter face à l'ampleur de l'offensive du patriarcat pour ramener les femmes à la raison et les enfermer à nouveau dans le carcan du paternalisme et de la dépendance.

Doit-on pour autant proposer aux femmes de se regrouper dans une organisation structurée de façon classique avec statuts et règlements, direction centralisée et calendrier d'opérations formalisés? Là où ce choix s'est traduit par la formation de partis féministes, cela semble avoir posé des problèmes menant soit à la scission ou à la disparition de la nouvelle formation politique - c'est le cas entre autres des partis féministes français et canadien - soit à l'isolement des femmes ainsi regroupées - parti féministe espagnol. Aucun de ces groupes n'a réussi à regrouper l'ensemble des groupes autonomes de femmes.

Par contre, nous assistons ici à d'autres formes de rassemblement plus modestes peut-être mais qui nous semblent plus prometteuses. C'est ainsi que les femmes qui ont quitté les anciennes organisations politiques marxistes-léninistes se rencontrent pour discuter entre elles ou se sont engagées dans des projets communs. 
D'autres groupes autonomes de femmes ont réussi à rassembler des centaines de femmes à l'occasion de colloques permettant de faire le point des activités dans leur secteur ou dans leur champ d'intervention pratique. C'est le cas du colloque sur la santé ou de celui sur la culture. Ces initiatives ne restent pas sans lendemain. Elles sont l'occasion de contacts fructueux, témoin la marche «seules sans peur la nuit». Les groupes luttant contre la pornographie ont mobilisé d'autres groupes de femmes et ainsi créé un réseau de solidarité qui fait que ce thème est devenu présent à l'intérieur des groupes qui se sont impliqués dans cette manifestation. D'autres groupes engagés dans des activités théâtrales ou la production de vidéo, livres ou revues sont en passe de se donner les moyens de survivre au processus d'érosion auquel les soumettent les divers organismes de subventions en joignant leurs efforts pour former un regroupement culturel ${ }^{33}$. Il est important aussi de noter que la Vie en rose, qui constitue un des ferments de ce type d'initiative, est elle-même un lieu de liaison privilégié pour faire connaître aux femmes l'ensemble des dimensions et la richesse de leurs luttes.

\section{Notre parole est politique}

Le féminisme comme courant politique n'a pas d'autres prétentions que de faire avancer la lutte des femmes et dénoncer toutes les institutions, comportements, attitudes patriarcales qui freinent les femmes dans la conquête de leur indépendance, leur autonomie, leur liberté et leur plaisir. Sa pratique diversifiée remet aussi en question une conception politique centralisée, un discours théorique abstrait, une analyse des rapports sociaux qui secondarise ou subordonne la question des femmes à des idéaux «supérieurs» ou plus «nobles». Mais cette pratique est aussi en

33. Entrevue réalisée avec Lise Moisan, membre du collectif de la V'ie en Rosé. juin 1983. 
train de tisser de nouveaux réseaux politiques de transformation sociale, qui pourraient dans certains cas aider la gauche à trouver des solutions à son incapacité actuelle d'inventer de nouvelles pratiques politiques qui mobilisent, enthousiasment tous ceux et celles qui vivent des rapports d'exploitation ou d'oppression. Le féminisme a réussi à percer non seulement parce qu'il s'est préoccupé de situations concrètes dans lesquelles les femmes se retrouvaient mais aussi parce qu'il a su diffuser partout autant dans Châtelaine, que dans Madame au foyer les résultats de ses enquêtes, de ses luttes dans un langage simple et accessible. Il ne s'est pas replié non plus sur des positions théoriques parce que ce qui est visé, c'est l'amélioration des conditions de vie et de travail des femmes. Le féminisme est sorti (ou presque!), et c'est là sa plus grande force, d'une vision hégémonique de la pratique politique. Il a permis l'expression d'une multitude de luttes, de points de vue parfois opposés mais qui contribuent par leur diversité à l'enrichir comme mouvement social. Son mode de fonctionnement par petits groupes, à partir de besoins concrets, avec comme principale ressource son énergie humaine et son esprit de créativité est devenu sa marque de fabrique. Par suite, son développement repose moins sur le sentiment d'urgence, défini par des contextes institutionnalisés. Ce qui le pousse de l'avant, c'est la volonté, la détermination de groupes de femmes de travailler ensemble à résoudre des problèmes qui leur tiennent à coeur tout en tenant compte de leurs capacités et de leur disponibilité réelles. Et aussi de leur désir de ne pas devenir des bêtes politiques. Parce que la vie est faite de réalités multiples... 